\title{
Stress and Infertility - The Chicken or the Egg
}

Rachel Donnison

Editorial Assistant

Citation: EMJ Repro Health. 2020;6[1]:20-22.

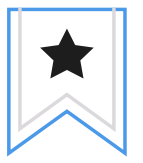

\begin{abstract}
- ORTY-SIX percent of people think stress or emotional distress causes fertility - problems, or the inability to achieve pregnancy with treatment. Stress, the response triggered from an imbalance between perceived threat and ability to cope with threat, was discussed in the session 'Stress and Infertility - The Chicken or the Egg' at the European Society of Human Reproduction and Embryology (ESHRE) virtual $36^{\text {th }}$ Annual Meeting, which combined the presentation by Prof Jacky Boivin, Cardiff University, Cardiff, UK, who made the case for stress causing lack of pregnancy, followed by Prof Angela Lawson, Northwestern University's Feinberg School of Medicine, Chicago, Illinois, USA, who argued the opposing case.
\end{abstract}

\section{"JUST RELAX AND IT WILL HAPPEN?" THE CASE FOR STRESS CAUSING LACK OF PREGNANCY}

Stress manifests itself in many forms, explained Prof Boivin: there is the stress of the pregnancy waiting period, work-related stress, and relationship stress. Prof Boivin began her session by taking the audience through her research journey; she has spent most of her career arguing that there is no direct association between stress and infertility. She updated the audience on what is known so far: no organism is advantaged from never reproducing, and therefore animals have evolved to reproduce despite highly stressful environments. For instance, citizens living in areas with significant stressors such as poverty, political warfare, and scarce resources, can still maintain some of the highest fertility rates in the world.

Claiming that the stress-induced infertility direct models are too simplistic to capture stress effects on fertility, Prof Boivin advocated for the creation of more complex models, posing the question: what factors should be in the stressfertility model, and what paths connect these factors to fertility?

\section{Stress-Induced Eating and Smoking}

Taking into account the actions that people undertake when they are stressed is a good start, commented Prof Boivin. Stress-induced eating is one such coping strategy, which typically leads to a higher-fat diet. This can become a barrier to fertility, as obesity is associated with poorer pregnancy outcomes in both females and males.

Smoking is another factor that may be induced by stress, and studies have shown that smokers have a shorter latency to smoke under stress, and a higher number of puffs is consumed. Smoking has long been associated with poorer outcomes in assisted reproductive technology (ART); it has been linked to reduced semen quality, lower levels of ovarian stimulation, and fewer oocytes.

\section{Behavioural Factors}

Behavioural indices related to ART treatment continuation include depression at the start of treatment, meaning that patients are less likely to take up treatment or take longer to do so. Prof Boivin quoted a large Danish cohort study of almost 40,000 participants, which found that individuals with depression were less likely to take up treatment and less likely to achieve a live birth. 
Stress-induced treatment drop-out is the final factor that Prof Boivin wished to include in the complex stress-infertility model. She relayed the results of a review which found that psychological distress in individuals made them five times more likely to discontinue treatment compared to people who had not experienced this. Prof Boivin was also careful to note that the depression could be a consequence of something else: "In fertility treatment, if couples are unsuccessful with treatment within the first 3 years... they are much more likely to have marital disruption," which can cause discontinuation of treatment and hence a lower chance of pregnancy.

Delayed decision-making is also a consequence of stress, which may be associated with reduced chances of pregnancy: "Comparing treatment when you're 39 to when you're 40, your chance of pregnancy drops substantially."

However, do we blame the patient? "No! Blame the poor access to affordable reproductive technologies and accumulated hardships and adversity that make trying to conceive so stressful."

Prof Boivin concluded that a new psycho-biobehavioural model of fertility in ART should question: "At what point does stress start to induce infertility?" such as in ovarian and testicular function or fertilisation. An appreciation of individual stress contributions by both females and males should also be included, as well as the plausible direct and indirect paths between stress and infertility and the time component used to capture the treatment journey.

\section{THE CASE AGAINST STRESS AS A CAUSE OF INFERTILITY}

\section{Consequence and Cause}

Providing the counterargument for stress and infertility, Prof Lawson began with the statistic that one in eight individuals struggle to conceive, and that this struggle is associated with psychological distress. This distress starts "before fertility treatment even begins, can worsen across failed treatment cycles, and can become so severe as to equal that of newly diagnosed cancer patients."

Prof Lawson believed that this distress originates from the assumption that it is easy to conceive, and that often females and males are told that it is their fault. Self-blame then leads to loss of control, and individuals try to control their lifestyle habits instead. When this does not work, an individual's anxiety increases as they start to worry that they will not become a parent, and this results in symptoms of bereavement and grief as they lose a sense of their identity.

\section{Biologic Plausibility}

We have two competing systems, explained Prof Lawson: the sympathetic, or fight-or-flight, and the parasympathetic, or feed-and-breed, 
nervous systems. The two systems vie for control of the hypothalamo-pituitary-gonadal (HPG) axis, which is the major neuroendocrine response system that controls the body's responses to stress. "There is a hypothetical shutdown of the HPG axis because of the release of stress hormones, cortisol and alpha-amylase, that may make it harder to get pregnant," continued Prof Lawson. However, research has shown that these systems never completely shut down as the body fights to maintain balance.

Choosing to back up her argument with metaanalyses instead of survey-based assessments, which she believed would be "cherry picking" of articles that are not representative of the entire literature, Prof Lawson conveyed the key take-home messages of several research studies on depression and anxiety, stress hormones, and relaxation.

\section{Depression and Anxiety}

The first article Prof Lawson evaluated showed no significant relationship between depression/ anxiety and chances of conceiving. The second, however, did find a link between clinical pregnancy rates and anxiety or stress levels, though most of the significance was only through small statistical effect sizes. Finally, the third study did find significant differences between patients with depression/anxiety and infertility, compared to controls. Each of the meta-analyses found different results, which Prof Lawson explained is because the authors chose which survey-based assessments they would include, and which they would not; there were methodological problems such as small sample sizes, heterogeneity in outcomes, different measures of psychological distress, and, perhaps the biggest problem, the lack of appropriate control variables. "One particularly important control variable is knowledge of one's prognosis," stressed Prof Lawson, as well as a diagnosis of polycystic ovary syndrome or endometriosis, which are independently associated with psychological distress; no studies have controlled for these variables to date.

\section{Stress Hormones and Infertility}

The two primary stress hormones are alphaamylase and cortisol. The research on cortisol is highly inconsistent, and the literature generally does not support a link to infertility. Conversely, the research on alpha-amylase has shown that in those who had higher levels of salivary alphaamylase, there was a slightly longer time to pregnancy after 5 months of trying to conceive. Despite this, in one particular study, nearly 90\% of the participants successfully conceived within the 12-month study period. These results confirmed what was already known about general populations and chances of conceiving: that many individuals will get pregnant within 6 months, and around $90 \%$ are successful within 12 months.

Prof Lawson suggested that this research has really been looking at changing hormones across menstrual cycles and the perimenopausal period. She stated that: "These changes in cortisol and alpha-amylase are associated with psychological distress, independent of changes in hormones."

\section{Research on Relaxation and Pregnancy Chances}

Additional meta-analysis data were then presented by Prof Lawson, this time on the topic of relaxation. Relaxing through psychotherapy was found to increase pregnancy chances, but only for patients who were not undergoing fertility treatment. Randomised controlled trials have failed to show a significant relationship between relaxation and likelihood of achieving pregnancy. Prof Lawson explained that the key take-away message here was that meta-analyses can be limited by the cherry picking of studies and can often include studies of poor quality; randomised controlled trials can be subject to selection and ascertainment bias.

Acupuncture is a relaxation method often talked about in terms of achieving pregnancy, but a 2013 Cochrane review showed no relationship between acupuncture and pregnancy chances. Yoga is another relaxation method, as well as music therapy, medical clowning, reflexology, and hypnosis; however, none of these have been tested in a randomised controlled trial.

In summary, Prof Lawson highlighted that overall, there has not been any evidence of a causal role in distress and infertility, unless one has functional hypothalamic amenorrhoea.

Despite their opposing views, Prof Boivin and Prof Lawson both agreed that the blame was never on the patient, and, in the words of Prof Lawson: "It is inappropriate to ever tell a patient to 'just relax."' 